\title{
Cancer Therapy: A Brief Outline
}

\author{
Smita Kayal ${ }^{1}$ \\ ${ }^{1}$ Department of Medical Oncology, Jawaharlal Institute of \\ Postgraduate Medical Education and Research, Pondicherry, \\ Puducherry, India
}

\begin{abstract}
Address for correspondence Smita Kayal, MD, DM, Department of Medical Oncology, Regional Cancer Centre, Jawaharlal Institute of Postgraduate Medical Education and Research, Dhanvantari Nagar, Puducherry 605006, India (e-mail: kayalsmita@gmail.com).
\end{abstract}

\begin{abstract}
Keywords

- cancer

- treatment

- review

Modern cancer treatment has evolved over several years to reach the current era of precision therapy. Exciting developments in all modalities of cancer treatment and rapidly growing arena of translational research are contributing to the steady improvement in clinical outcomes. Although several old and new challenges have to be overcome, parallel technological advances in the tools and techniques of drug discovery has promise for future. An outline of the overall approach to cancer management and a broad perspective of multimodality treatment methods are discussed in this brief review.
\end{abstract}

\section{Introduction}

History of modern cancer treatment dates back to about 200 years, although cancer is as old as humankind or even life. ${ }^{1-3}$ The incidence rate for all cancers in all age groups combined is progressively rising, from 182.3 per 100,000 in 2012 to 197.9 per 100,000 in 2018 globally. Nevertheless, mortality rates overall have been marginally but steadily declining over the past few decades, from 102.4 per 100,000 in 2012 to 101.1 per 100,000 in $2018 .{ }^{4}$ GLOBOCAN 2018 estimated an incidence of 18.1 million new cancer cases and 9.6 million cancer deaths worldwide for $2018 .{ }^{4}$ Growing understanding of cancer biology, parallel advances in diagnosis and risk stratification, improved cancer treatment modalities, new drug discoveries and better supportive care, and cooperative group trials, all have resulted in significant rise of survival for both childhood and adult cancers. In this very brief review on cancer therapy, we attempt to summarize the principles of cancer treatment and their application and challenges in clinical practice for the beginners in oncology.

\section{Cancer Treatment Modalities}

Cancer is broadly divided into solid tumors and hematological malignancies. The intent of cancer therapy may be curative or palliative depending on the disease and patient characteristics. Solid tumors of different organs are generally staged as localized, locoregional, or metastatic disease. Localized and regional solid tumors are primarily treated with locoregional treatment modalities, like surgery and radiotherapy. Also, depending on the stage and disease extent, systemic chemotherapy is added as adjunct to local treatment to prevent recurrences and thereby improve survival. Metastatic solid tumors and all hematological malignancies are principally treated with systemic chemotherapy. Locoregional treatment with surgery or radiotherapy is also considered for certain metastatic solid tumors (e.g., germ cell tumor, colorectal cancer, renal cell carcinoma, pediatric solid tumors) and hematological malignancies (e.g., lymphomas with bulky or residual disease, plasmacytoma) as component of main treatment plan or for palliation. Other components of systemic therapy include hormone therapy, various targeted agents, monoclonal antibodies, and immunotherapy which are used in the course of treatment of different solid and hematological malignancies. Thus, treatment of cancer generally requires multimodality approach which has to be tailored as per the cancer type, stage, and biology, and according to the patient's clinical risk group and demographic characteristics. - Fig. 1 summarizes the available modalities and multimodal approach to cancer treatment.

\section{Locoregional Therapy}

Earliest treatment of cancer in the 1800s for patients with localized tumor growths consisted of radical anatomical dissection based on Halstedian concepts of orderly contiguous tumor spread and consideration of cancer as locoregional disease autonomous of its host. In early 1900s, radiotherapy emerged as a modality of cancer cell kill through 


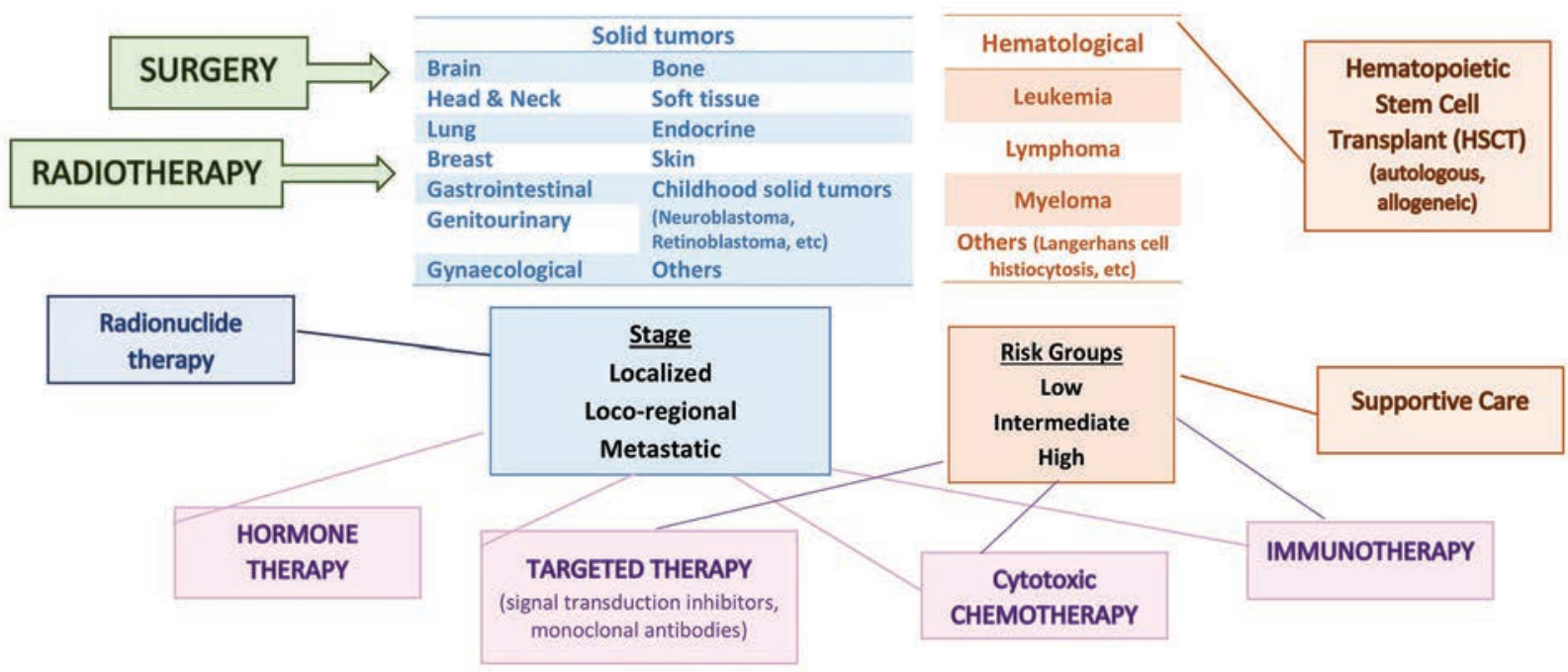

Fig. 1 Cancer treatment modalities: Locoregional treatment modalities such as surgery and radiotherapy are used to treat early stage solid tumors. Systemic therapy such as cytotoxic chemotherapy, hormone therapy, targeted drugs, and immunotherapy are used alone or in different combinations to treat hematological malignancies, as adjunct therapy for early or locally advanced solid tumors and as palliative therapy for metastatic solid tumors. Hematopoietic stem cell transplant is used for various indications in the treatment of hematological malignancies. Advances in supportive care have supplemented the administration of all these intense treatment modalities.

ionizing radiation that disrupts various pathways of cell cycle. ${ }^{5}$ Radiotherapy thus provided an alternative or adjunct modality of locoregional treatment for various solid tumors. In the past two to three decades, significant technological advances in the conduct of surgery (e.g., endoscopic surgery, laparoscopic surgery, robotic surgery) and delivery of radiotherapy (e.g., intensity-modulated radiotherapy, volumetric modulated arc therapy, stereotactic body radiotherapy) have led to more precision in locoregional treatment, more organ preservation methods, and reduced morbidity. Parallel advances in reconstructive surgeries and various rehabilitation procedures have improved quality of life for patients with early stage solid tumors.

\section{Systemic Therapy}

Systemic anticancer treatment started with the discovery of cytotoxic chemotherapy in the late 1940s, the first few drugs being nitrogen mustard compounds and antifolates used in the treatment of leukemias and lymphomas. Since then, from 1949 to 2014, a total of 150 medicines has been approved including cytotoxic drugs and targeted agents with an indication for at least one type of cancer. ${ }^{6}$ Most of the cytotoxic drugs are alkylating agents, antimicrotubule agents, antimetabolites, and topoisomerase inhibitors which work in different phases of cell cycle, while most of the targeted drugs belong to signal transduction inhibitors, gene expression modulators, apoptosis inducers, hormone therapies, angiogenesis inhibitors, immune modulators, and monoclonal antibodies which targets one or more of the hallmarks of cancer pathogenesis. In the past 5 years from 2015 to 2019, approximately 60 new anticancer medicines, latest being the immunotherapy group of drugs, have been approved and several older drugs are being approved for newer indications, underscoring the steadily escalating efforts in drug discovery and translational cancer research. - Fig. 2 summarizes the major classes of cytotoxic drugs and targeted agents. Additionally, several supportive care drugs used to treat various side effects of cancer therapy as nausea and vomiting, myelosuppression, febrile neutropenia, gastrointestinal toxicities, neuropathy, and others have developed in parallel, allowing for timely and adequate delivery of intensive treatment protocols. Systemic chemotherapy is administered as cycles or periodic courses, with interval between two doses of an average 3 to 4 weeks, to allow adequate time for normal cells to recover from collateral cytotoxicity.

Combination therapy with enterally or parenterally administered cytotoxic drugs with different mechanisms of action and differing dose limiting toxicities forms the mainstay of treatment of hematological malignancies. Optimization of drug dose, regimen, and schedule over decades through conduct of cooperative group trials have led to significant cure rates in acute leukemias and lymphomas and prolonged progression-free survival in myeloma. Novel cytotoxic drugs, targeted agents, and monoclonal antibodies are used either as single agent or in combination for treatment of relapsed/refractory diseases and for particular indications have moved to the first line therapy (e.g., rituximab in B cell lymphomas). A few targeted agents have changed the treatment paradigm of some diseases, for example, imatinib, a tyrosine kinase inhibitor targeting $B C R-A B L$, introduced in 2001 in the treatment of chronic myeloid leukemia have obviated the need for upfront allogeneic stem cell transplant in this disease. Similarly, all-trans-retinoic acid and arsenic trioxide targeting and releasing the differentiation block in acute promyelocytic leukemia caused by the PML-RARA translocation have resulted in cure rates of 80 to $90 \%$ with a chemotherapy-free protocol. 


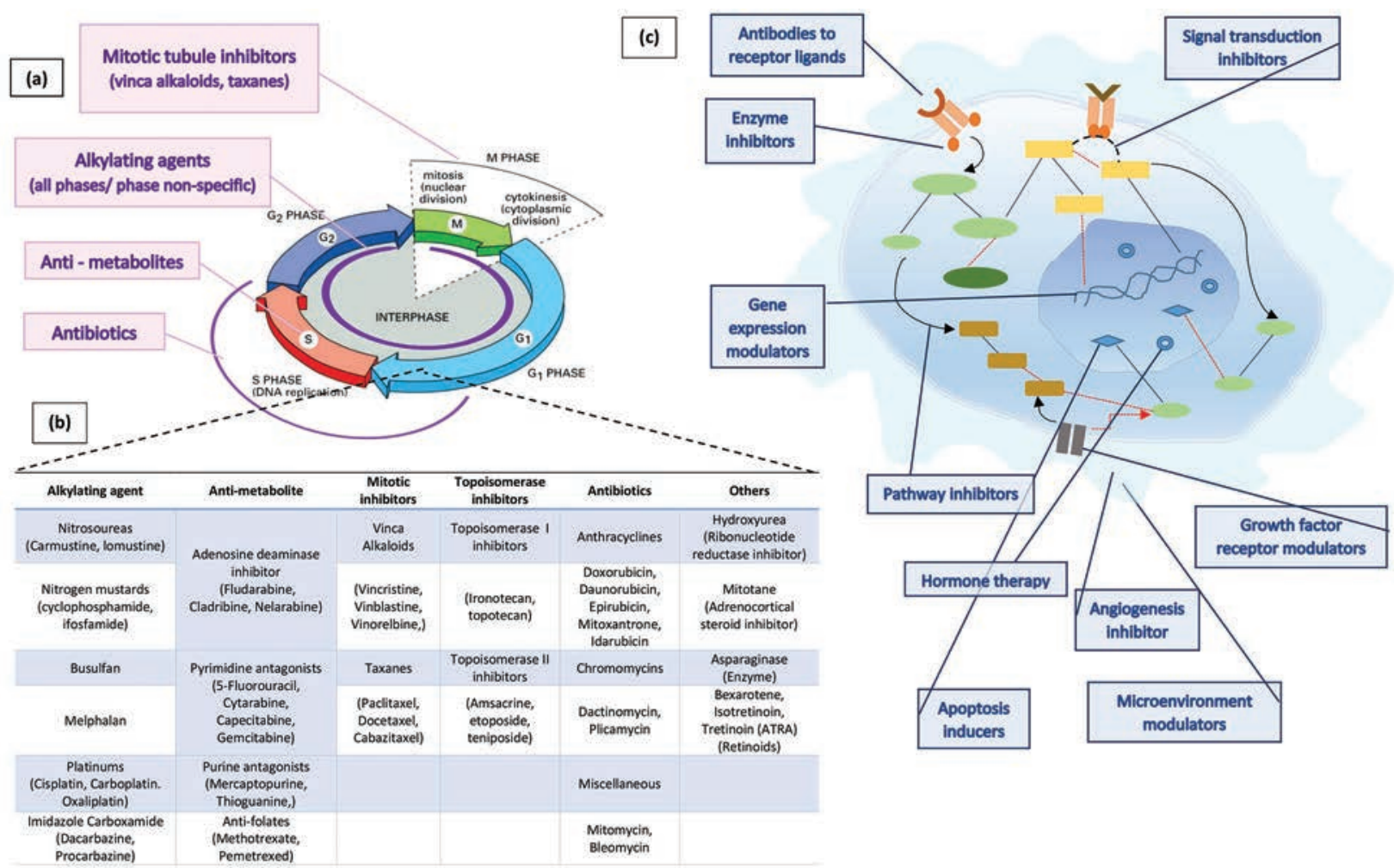

Fig. 2 (A) Cytotoxic class of drugs acting on different phases of cell cycles. (B) Table illustrating the major classes and components of cytotoxic chemotherapy. (C) Simplified illustration of families of targeted agents and their respective subcellular targets in the cell, nucleus, and microenvironment.

In solid tumors, the evolving concept of operable cancer being systemic disease with potential for dissemination through lymphatics and blood stream even in early stages and recognition of complex host-tumor interrelationship affecting disease biology, which were contrary to the old Halstedian principles, led to the experiments for adjuvant systemic therapy in the treatment of localized disease. These experiments of systemic therapy as adjunct to surgery led by Fisher and colleagues in the 1970s, concluded that two paradigms govern the management of cancer, first is related to the use of surgery to eradicate local and regional disease; the second is related to the eradication of systemic disease (micrometastases). ${ }^{7}$ The treatment of patients who has no identifiable metastatic disease with systemic adjuvant therapy (after surgery) or neoadjuvant therapy (before surgery) with either hormonal agents (e.g., tamoxifen in breast cancer), targeted or cytotoxic chemotherapy, or both have resulted in decreased local and regional recurrences as well as distant metastases after minimal conservative surgery and have improved survival in patients of various solid tumors to the tune of 4 to $15 \%$ absolute benefit at different stages.

Chemotherapy, along with locoregional therapy, is also an integral component of curative treatment of certain metastatic solid tumors such as germ cell tumors, choriocarcinoma, and neuroblastoma, which are highly chemo-sensitive. For most of the other solid tumors with advanced and metastatic disease, systemic chemotherapy and targeted agents are used for palliative treatment. However, with the advent of combination chemotherapy, targeted agents, monoclonal antibodies, and immunotherapy, several sequential lines of treatment can be administered safely even for advanced diseases with resultant improvement in clinical outcomes for many of the common malignancies such as breast, prostate, lung, and colorectal cancers. Yet, treatment intent still remains palliative and not curative in majority of metastatic solid tumors, nevertheless, provides better quality of life and considerable prolongation of survival.

\section{Multimodality Approach}

Multidrug, multiphase combination chemotherapy regimens comprising of cytotoxic drugs, targeted agents, monoclonal antibodies, etc. in defined schedule forms the basis of treatment of hematological cancers. Radiotherapy is generally given for bulky or residual disease sites in lymphoma, for prophylactic or therapeutic cranial irradiation in leukemias, and as single modality radical treatment for plasmacytoma. - Table 1 shows an outline of some of the most widely used treatment protocols for some common hematological malignancies. Hematopoietic stem cell transplant (HSCT) either autologous or allogeneic is used for consolidation treatment as part of frontline therapy in certain high-risk hematological malignancies, for example, multiple myeloma (autologous HSCT), Philadelphia positive acute lymphoblastic leukemia in adults (allogeneic HSCT), intermediate and high-risk acute myeloid leukemia (allogeneic HSCT), and for salvage treatment of refractory/ relapsed hematological cancers. 
Table 1 Common chemotherapy regimens used in hematological malignancies

\begin{tabular}{|l|l|}
\hline Disease & Common chemotherapy regimens \\
\hline $\begin{array}{l}\text { Hodgkin's } \\
\text { lymphoma }\end{array}$ & $\begin{array}{l}\text { ABVD (adriamycin, bleomycin, } \\
\text { vinblastine, dacarbazine) }\end{array}$ \\
\hline $\begin{array}{l}\text { Non-Hodgkin's } \\
\text { lymphoma }\end{array}$ & $\begin{array}{l}\text { CHOP } \pm \text { R (cyclophosphamide, doxorubicin, } \\
\text { vincristine, prednisone) / Rituximab }\end{array}$ \\
\hline $\begin{array}{l}\text { Multiple } \\
\text { myeloma }\end{array}$ & $\begin{array}{l}\text { VRD (bortezomib, lenalidomide, } \\
\text { dexamethasone) }\end{array}$ \\
\hline $\begin{array}{l}\text { Acute myeloid } \\
\text { leukemia }\end{array}$ & 3+7 (daunorubicin and cytarabine) \\
\hline $\begin{array}{l}\text { Acute } \\
\text { promyelocytic } \\
\text { leukemia }\end{array}$ & $\begin{array}{l}\text { ATRA + ATO (all-trans-retinoic acid, } \\
\text { arsenic trioxide) }\end{array}$ \\
\hline $\begin{array}{l}\text { Acute lym- } \\
\text { phoblastic } \\
\text { leukemia }\end{array}$ & $\begin{array}{l}\text { Intensive multiagent chemotherapy (steroid, } \\
\text { 6-MP, vincristine, daunorubicin L-asparagi- } \\
\text { nase, cytarabine, cyclophosphamide, high } \\
\text { dose methotrexate) in induction and consol- } \\
\text { idation phase followed by maintenance with } \\
\text { 6-MP and methotrexate }\end{array}$ \\
\hline $\begin{array}{l}\text { Chronic } \\
\text { lymphocytic } \\
\text { leukemia }\end{array}$ & BRendamustine, rituximab) \\
\hline $\begin{array}{l}\text { Chronic } \\
\text { myeloid } \\
\text { leukemia }\end{array}$ & $\begin{array}{l}\text { Imatinib (BCR-ABL tyrosine kinase } \\
\text { inhibitor) }\end{array}$ \\
\hline
\end{tabular}

In solid tumors, depending on the stage, all the three main modalities-surgery, radiotherapy, and chemotherapy-are used in the frontline treatment. Further, hormonal therapy, targeted agents are added to the protocol in certain tumors depending on the biological characteristics and risk group. We will discuss two tumors-breast carcinoma and neuroblastoma (common childhood tumor)-as prototype for multimodality treatment plan. - Fig. 3 highlights the usual treatment modalities and course for breast carcinoma and neuroblastoma. For stage II/III breast carcinoma, general course of treatment includes neoadjuvant combination chemotherapy (mainly with anthracyclines and taxanes) followed by surgery (either breast conservation or mastectomy depending on the baseline stage) and followed by radiotherapy (depending on type of surgery and baseline stage). Hormonal therapy for a duration of 5 to 10 years is added for patients with estrogen or progesterone receptor positive tumors and anti-Her 2 therapy (monoclonal antibody directed at the epidermal growth factor receptor Her 2) for Her 2 positive tumors. For a subset of very early stage, hormone receptor positive, Her 2 negative tumors, with low recurrence score by molecular tests treatment can be done by only surgery followed by hormonal therapy without the need for radio- or chemotherapy. In high-risk metastatic neuroblastoma, treatment is done with all modalities as combination chemotherapy, surgery, autologous HSCT, radiotherapy, and posttransplant maintenance treatment with differentiation agent (isotretinoin) and immune modulators (anti-GD2 antibody and interleukin-2). Thus, majority of the malignancies require a multimodality treatment approach for curative outcomes. Treatment decisions are generally taken in a multidisciplinary tumor board consisting of surgeons, anesthetist, radiation oncologist, and medical oncology experts. Further, the multidisciplinary team should also consist of nutritionist, physiotherapist, speech therapist, palliative care physicians, infection disease expert, psychosocial counselors, and other specialists (endocrinologist, cardiologist, etc., depending on the age, cancer, and treatment type) for guiding supportive care during the course of treatment, rehabilitation posttreatment, and for monitoring and management of late side effects.

In metastatic solid tumors, for most of the common malignancies of lung, breast, prostate, colorectal, renal, ovary, etc., a multitude of treatment options are now available for the first line and subsequent lines of therapy that have resulted in a significant increase in overall survival, up to 12 to 18 months on average over historical outcomes, in particular patient subsets in these cancer subtypes. These treatment options include besides conventional cytotoxic chemotherapy, targeted therapy related to the specific driver genomic alteration, hormonal therapy for hormonally driven cancers, drugs targeting the angiogenesis pathway and tumor microenvironment, and immunotherapy targeting the immune checkpoints involved in tumor cell to immune cell interactions. - Table 2 outlines the common therapies currently available for metastatic castrate-resistant prostate cancer which can be ordered in numerous schedules for sequential use. Current challenge in management of these metastatic solid tumors is in optimizing the right combination and sequencing of treatment. Another important clinical challenge is in evaluating cost effectiveness of the newer drugs for palliative treatment and in identifying futility of further treatment or when to stop further treatment for patients with poor general condition and progressive disease.

Other aspects of cancer care such as prevention, screening, early diagnosis, toxicity management, and rehabilitation are important areas, but are beyond the scope of the current review. Discussing the details on each cancer modality and drug, and treatment for individual cancers, is also outside the space of this brief summary. Many comprehensive international guidelines are available that summarizes the treatment approach and algorithm for management of all common malignancies and serve as useful resource. ${ }^{8,9}$ Finally, in clinical practice, treatment decisions require the expertise and experience of the oncology team.

\section{Treatment Adaptation: Based on Prognostic and Predictive Biomarkers}

Prognostic factor is defined as measurement taken at the time of diagnosis or treatment that is associated with the outcome, determining a patient's ability to fare in the absence of treatment, for example, age of the patient, stage determined by tumor size, nodal involvement and distant spread, grade, cytogenetic or molecular profile, etc. Often a combination of clinical pathological and genetic changes are taken together to determine risk groups and based on the individual risk group treatment can be tailored-either intensified for high-risk group patients or de-intensified for low risk. 


\begin{tabular}{|c|c|}
\hline $\begin{array}{l}52 \mathrm{yr} / \mathrm{F} \\
\text { Left } \\
\text { breast } \\
\text { mass }\end{array}$ & $\begin{array}{l}\text { Clinical factors } \\
\text { - Age } \\
\text { - Tumor Size } \\
\text { - Node positivity } \\
\text { - Margins }\end{array}$ \\
\hline \multicolumn{2}{|c|}{$\begin{array}{l}\text { Biology } \\
\text { - ER/PR (estrogen } \\
\text { \& progesterone receptor) } \\
\text { - HER } 2 \text { neu amplification } \\
\text { - Proliferation } \\
\text { - Grade } \\
\text { - LVSI (Lymphovascular invasion) }\end{array}$} \\
\hline
\end{tabular}
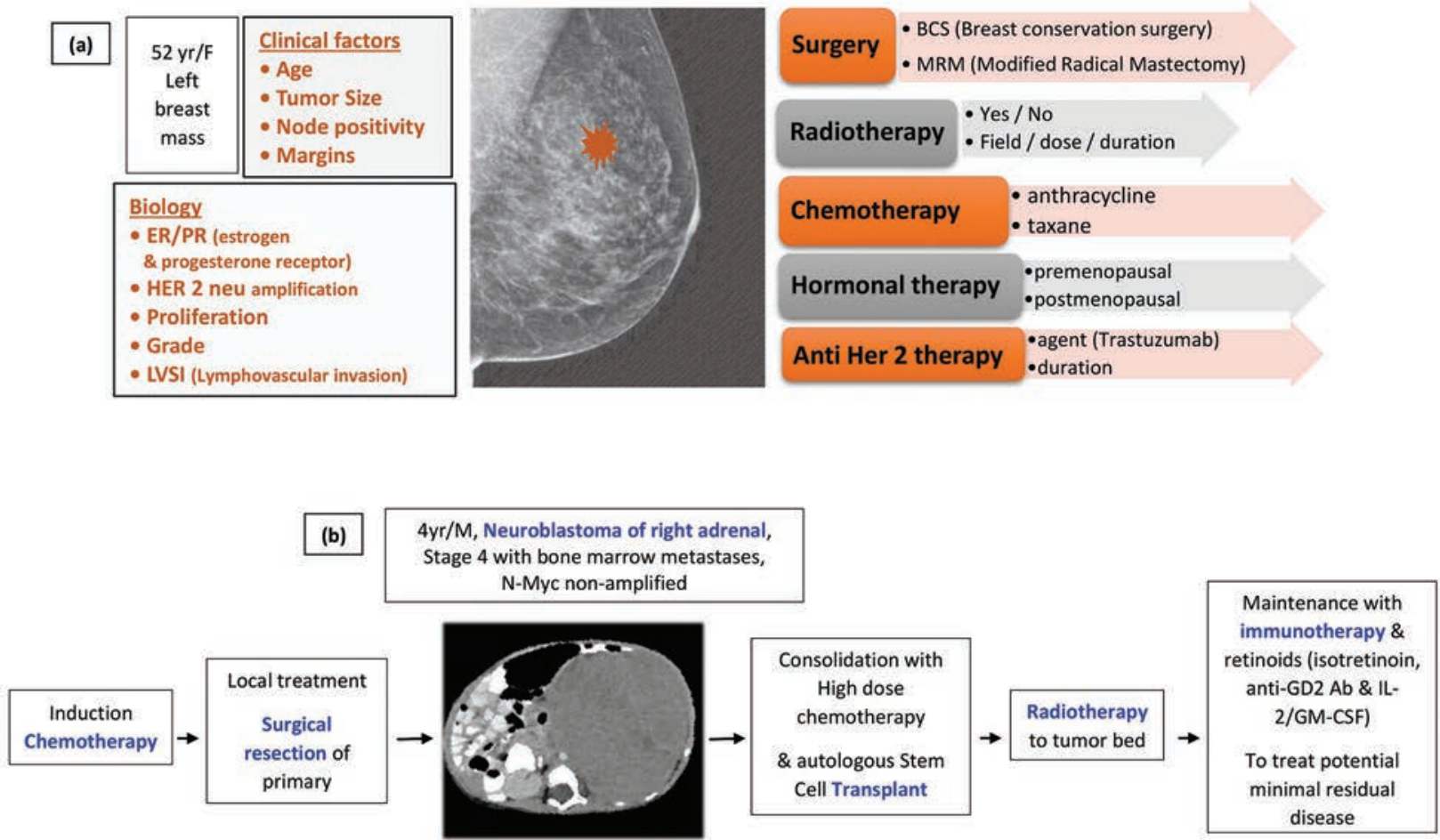

Fig. 3 Multimodality treatment approach for solid tumors, two prototype examples: (A) Breast carcinoma, depending on the clinical and pathological factors, locoregional and systemic treatment are used to different extent and combination. (B) Neuroblastoma, for high-risk patients all treatment modalities of combination chemotherapy, surgery, radiotherapy, autologous stem cell transplant, immunotherapy, and differentiation agent are used in sequence.

Table 2 Treatment options for metastatic castrate resistant prostate cancer: clinical conundrum

\begin{tabular}{|c|c|c|c|c|}
\hline $\begin{array}{l}\text { Drug (chemotherapy, } \\
\text { hormonal therapy, } \\
\text { immunotherapy, radio-ligand } \\
\text { therapy) }\end{array}$ & First line options & $\begin{array}{l}\text { Second line } \\
\text { options }\end{array}$ & $\begin{array}{l}\text { Third line } \\
\text { options }\end{array}$ & $\begin{array}{l}\text { Third line: Beyond and novel } \\
\text { agents }\end{array}$ \\
\hline Abiraterone & \multirow[t]{2}{*}{ Sipuleucel-T } & \multirow[t]{2}{*}{ Abiraterone } & \multirow[t]{2}{*}{ Cabazitaxel } & ${ }^{177}$ Lu-PSMA therapy \\
\hline Enzalutamide & & & & Pembrolizumab \\
\hline Docetaxel & \multirow[t]{2}{*}{ Abiraterone } & \multirow[t]{2}{*}{ Sipuleucel-T } & \multirow[t]{2}{*}{ Radium 223} & $\begin{array}{l}\text { PARP (poly ADP ribose poly- } \\
\text { merase) inhibitors }\end{array}$ \\
\hline Cabazitaxel & & & & VEGF inhibitors \\
\hline Sipuleucel-T & \multirow[t]{2}{*}{ Enzalutamide } & \multirow[t]{2}{*}{ Docetaxel } & \multirow[t]{2}{*}{ Enzalutamide } & Src inhibitors \\
\hline Radium 223 & & & & HSP90 inhibitors \\
\hline${ }^{177}$ Lu-PSMA therapy & \multirow[t]{3}{*}{ Docetaxel } & \multirow[t]{2}{*}{ Enzalutamide } & \multirow[t]{3}{*}{ Abiraterone } & AKT inhibitors \\
\hline \multirow{2}{*}{$\begin{array}{l}\text { Pembrolizumab (for MSI high } \\
\text { tumors) }\end{array}$} & & & & PI3 kinase inhibitors \\
\hline & & Radium 223 & & MTOR inhibitors \\
\hline
\end{tabular}

Predictive factor is a measurement that predicts response or lack of response to a specific treatment. Some common examples include epidermal growth factor receptor (EGFR) mutation in lung cancer that determines response to EGFR inhibitor like gefitinib, Her2/neu amplification in breast cancer that determines response to anti-Her2 therapy like trastuzumab or lapatinib, and K-RAS/N-RAS mutations in colorectal cancer which are a negative predictive factor for response to EGFR monoclonal antibody such as cetuximab. Several other biomarkers (mutations or polymorphism) either in the tumor genome or in germline deoxyribonucleic acid involving the drug metabolizing pathways are known that can affect responses to particular drugs. ${ }^{10,11} \mathrm{~A}$ plethora of biomarkers have been studied in different diseases but clinical utility has been established only for a handful of them. With growing understanding of cancer biology and disease pathogenesis, molecular classification is evolving for all cancer types which when clinically validated will help in further prognostication and identification of predictive markers and thereby in personalizing cancer treatment. 


\section{Clinical Endpoints}

Treatment results in oncology are generally defined by response rates, disease-free remission, morbidity or late sequel of treatment, quality of life, and survival. In other words, a clinically relevant endpoint is a characteristic that reflects how a patient feels, functions, or survives. In cancer patients, the risk for death from a specific neoplasm is highest in the initial years after diagnosis; it decreases progressively thereafter. To apply the word "cured," the time from the cancer diagnosis must be such that the patient's risk of death does not, because of cancer, exceed that of a sex- and age-matched general population. ${ }^{12,13}$ However, in oncology, use of word "cure" is debatable in view of late and very late relapses in certain malignancies and the more commonly used terminology is long-term survivor. For some of the early stage and good risk malignancies, for example, testicular germ cell tumor, thyroid cancer, Hodgkin's lymphoma, childhood acute lymphoblastic leukemia, and gestational trophoblastic neoplasm, 5- and 10-year disease-free survival (that is the period the disease remains in continuous clinical remission and without any recurrence) is close to 85 to $90 \%$ which may be taken as functional "cure." For other tumors, available treatment modalities have prolonged survival with a fairly better quality of life making many cancers a chronic disease. Ongoing translational research in cancer biology and treatment may further help to improve their outcomes.

\section{Challenges and Future of Cancer Treatment}

Cancer therapy is a continuously evolving field and every year there is considerable upsurge in new drug discovery and approvals, in drug repurposing and approval of newer indications for older drugs, in newer methods of drug delivery and optimized management of toxicities, in discovery of new predictive biomarkers and new treatment approaches, and also in technological advances in locoregional treatment modalities of surgery and radiotherapy. However, there remain several challenges in the path to translational of all these new developments in practice of real precision medicine and into clinically meaningful benefit in cancer survival. Some of the important challenges are dealing with tumor heterogeneity, handling drug resistance either due to pharmacogenomics differences in drug metabolism and transport or more commonly due to acquired mutations/alterations in cancer genome or its downstream pathway, finding of actionable alterations in the tumor tissue or its microenvironment (currently druggable genomic alterations represent only a small subset in certain cancer types), and identification and validation of predictive markers of immune therapy. Also, the disconcerting background of genomic variability creates issues regarding clinical interpretation, application, and validation of enormous and complex genomic data. Another practical challenge is in finding the optimal combination regimen, targeting several molecular alterations concurrently or in precise sequence, and validating them in clinical trials for demonstrating final benefit in survival. ${ }^{14}$
Some of the additional challenges pertaining to resource-limited settings in the Indian context are wide disparity in the access to cancer treatment, delayed presentation with higher disease burden, heterogeneity in resources, available expertise and treatment cost and payment structure across centers, poor social support system, significant financial constraints as most of the treatment expenses are met out of pocket, poor understanding of the disease and its treatment course and consequently higher treatment abandonment rates, restrictive access, nonavailability or prohibitive cost of the latest anticancer drugs, and very low rates of recruitment into well-designed clinical trials.

Besides development of newer generations of older targeted agents, discovery of new drugs targeting single molecular abnormality or pathway, and the expanding field of immune checkpoint inhibitors, the following novel approaches to cancer treatment which have already been studied in early phase clinical trials are making headway into mainstream therapy. These include cellular immune therapy such as CAR-T cell (chimeric antigen receptor) therapy, anticancer vaccines, and new therapeutic approaches based on genomic editing. ${ }^{15}$ Also, so far the approach to cancer treatment had been reductionist, which is targeting single molecular abnormality or cancer pathway that have modestly improved outcomes, but to move toward potential cure, systems biology or multipronged approach targeting several driver molecular pathways or cancer hallmarks of etiopathogenesis simultaneously might be a promising therapeutic strategy. ${ }^{14}$ As mentioned above, challenge for this approach is in optimizing the right combination or sequence, and in finding valid biomarkers; however, with better comprehension of next-generation precision oncology tools and data this should be attainable in the near future. Further, to find answer to locally relevant clinical problems in the Indian context, well-designed clinical trials through multicenter collaboration at the regional or national level is a pressing need that would be vital to improve outcomes close to that seen in the western developed world.

\section{Conclusion}

In this concise review, we have attempted to outline the major modalities of treatment in oncology, their evolution in brief, recent advances and challenges, and multimodality approach to cancer management in clinical practice with some common examples. Overall, treatment has to be evidence- and value-based, cost effective, and guided by local problems, expertise, and resources. We hope this would be a useful summary on cancer therapy for a new induct into oncology or for anybody who is keen on understanding the basic principles of cancer treatment, and encourage them to read and explore further and contribute their bit to cancer management.

\section{Conflict of Interest}

None declared. 


\section{References}

1 DeVita VT, Jr, Rosenberg SA. Two hundred years of cancer research. N Engl J Med 2012;366(23):2207-2214

2 Arruebo M, Vilaboa N, Sáez-Gutierrez B, et al. Assessment of the evolution of cancer treatment therapies. Cancers (Basel) 2011;3(3):3279-3330

3 Sudhakar A. History of cancer, ancient and modern treatment methods. J Cancer Sci Ther 2009;1(2):1-4

4 Bray F, Ferlay J, Soerjomataram I, Siegel RL, Torre LA, Jemal A. Global cancer statistics 2018: GLOBOCAN estimates of incidence and mortality worldwide for 36 cancers in 185 countries. CA Cancer J Clin 2018;68(6):394-424

5 Connell PP, Hellman S. Advances in radiotherapy and implications for the next century: a historical perspective. Cancer Res 2009;69(2):383-392

6 Sun J, Wei Q, Zhou Y, Wang J, Liu Q, Xu H. A systematic analysis of FDA-approved anticancer drugs. BMC Syst Biol 2017;11(Suppl 5):87

7 Fisher B. Biological research in the evolution of cancer surgery: a personal perspective. Cancer Res 2008;68(24):10007-10020

8 NCCN Clinical Practice Guidelines in Oncology (NCCN Guidelines) with NCCN Evidence Blocks [Internet]. Available at: https://www.nccn.org/evidenceblocks/default.aspx. Accessed September 18, 2019
9 Oncology Clinical Practice Guidelines | ESMO [Internet]. Available at: https://www.esmo.org/Guidelines. Accessed September 18, 2019

10 Lauschke VM, Milani L, Ingelman-Sundberg M. Pharmacogenomic biomarkers for improved drug therapy-recent progress and future developments. AAPS J 2017;20(1):4

11 Concetta Crisafulli C, Romeo PD, Calabrò M, Epasto LM, Alberti S. Pharmacogenetic and pharmacogenomic discovery strategies. Cancer Drug Resist 2019;2:225-241

12 Baade PD, Youlden DR, Chambers SK. When do I know I am cured? Using conditional estimates to provide better information about cancer survival prospects. Med J Aust 2011;194(2):73-77

13 Tralongo P, Maso LD, Surbone A, et al. Use of the word "cured" for cancer patients-implications for patients and physicians: the Siracusa charter. Curr Oncol 2015;22(1):e38-e40

14 Zugazagoitia J, Guedes C, Ponce S. Ferrer I, Molina-Pinelo S, Paz-Ares L. Current challenges in cancer treatment. Clin Ther 2016;38(7):1551-1566

15 Falzone L, Salomone S, Libra M. Evolution of cancer pharmacological treatments at the turn of the third millennium. Front Pharmacol 2018;9:1300 